\title{
MiR-367 alleviates inflammatory injury of microglia by promoting M2 polarization via targeting CEBPA
}

\author{
Hui Pei ${ }^{1}$ - Qian Peng ${ }^{2}$. Shewei Guo ${ }^{3}$ Y Yulei Gu ${ }^{1} \cdot$ Tongwen Sun $^{4} \cdot$ Dong Xu ${ }^{1}$. Yumin Jiang ${ }^{1} \cdot$ Jiafeng Xie ${ }^{1}$. \\ Luanluan Zhang ${ }^{1} \cdot$ Zhiqiang Zhu $^{1}$ (D)
}

Received: 14 February 2020 / Accepted: 1 October 2020 / Published online: 4 November 2020 / Editor: Tetsuji Okamoto

(C) The Author(s) 2020

\begin{abstract}
MiR-367 was reported to regulate inflammatory response of microglia. CCAAT/enhancer-binding protein $\alpha$ (C/EBPA) could mediate microglia polarization. In this study, we explored the possible roles of miR-367 and CEBPA in intracerebral hemorrhage (ICH). ICH and normal specimens were obtained from the tissue adjacent to and distant from hematoma of ICH patients, respectively. Microglia were isolated and identified by immunofluorescence. The isolated microglia were treated with erythrocyte lysate and randomly divided into 8 groups using different transfection reagents. The transfection efficiency of miR-367 was determined by qRT-PCR. The expressions of M1 and M2 microglia markers were detected by Western blotting. The relationship between CEBPA and miR-367 was confirmed by dual luciferase reporter system. Flow cytometry was performed to determine the level of apoptosis in the cells transfected with miR-367 and CEBPA in erythrocyte lysate-treated microglia. We found that miR367 expression level was downregulated in ICH specimens. Erythrocyte lysate-treated microglia was successfully established using erythrocyte lysate, as decreased miR-367 expression was observed. Overexpression of miR-367 could significantly decrease the expressions of MHC-II, IL-1 $\beta$, and Bax, reduced apoptosis rate, and increased the expressions of CD206, Bal-2, and Arg- 1 in erythrocyte lysate-treated microglia. CEBPA was proved to be a direct target for miR-367, which could inhibit microglia M2 polarization and increase apoptosis rate. However, in the presence of both CEBPA and miR-367 mimic, the protein and mRNA expressions of CEBPA were decreased, leading to promoted microglia M2 polarization and a decreased apoptosis rate. MiR-367 regulates microglia polarization by targeting CEBPA and is expected to alleviate ICH-induced inflammatory injury.
\end{abstract}

Keywords miR-367 $\cdot$ M2 polarization $\cdot$ Microglia $\cdot$ CEBPA $\cdot$ Intracerebral hemorrhage

\section{Introduction}

Hemorrhagic stroke (also known as intracerebral hemorrhage $(\mathrm{ICH}))$ is the most acute and serious cerebrovascular disease,

Hui Pei and Qian Peng contributed equally to this work.

Zhiqiang Zhu

zhiqinagz_zzq@163.com

1 Department of Emergency Intensive Care Unit, the First Affiliated Hospital of Zhengzhou University, No.1, East Jianshe Road, Erqi District, Zhengzhou 450052, Henan Province, China

2 Department of Plastic Surgery, The First Affiliated Hospital of Zhengzhou University, Zhengzhou, China

3 Department of Neurosurgery, The First Affiliated Hospital of Zhengzhou University, Zhengzhou, China

4 Department of General Intensive Care Unit, The First Affiliated Hospital of Zhengzhou University, Zhengzhou, China as it has a quick onset and high mortality and disability rates (Shi et al. 2016; Zhou et al. 2017) and can cause hematoma and secondary pathological processes (Psaila et al. 2009; van Asch et al. 2010; Tatlisumak et al. 2018). ICH causes secondary injury via various pathways, of which inflammatory response is one of the most pivotal pathways (Hamzei Taj et al. 2016). Therefore, inhibiting the production of proinflammatory mediators is possibly an effective strategy for preventing brain injury after ICH.

Microglia plays an important role in inflammatory response (Shi et al. 2016). After ICH, microglia is activated and release inflammatory factors, thereby exacerbating ICHinduced injury (Zhang et al. 2017). Increasing evidence showed that microglia with different phenotypes can produce either detrimental or beneficial responses depending on specific environmental signals (Boche et al. 2013; Lee et al. 2016; Shi et al. 2016; Zhang et al. 2017). Microglia can be divided into classically activated M1 and alternatively activated M2 according to their surface markers and intracellular 
cytokines (Boche et al. 2013; Lee et al. 2016; Shi et al. 2016). Classically activated M1 can increase pro-inflammatory cytokines (e.g., IL-1 $\beta$, TNF- $\alpha$, iNOS) and M1 marker major histocompatibility complex class II (MHC-II) and aggravate inflammatory response, while alternatively activated M2 can secrete anti-inflammatory cytokines (e.g., Arg-1, IL-4, IL-13) and M2 marker CD206 and exerts an opposite effect to that of M1 microglia (Miron et al. 2013; Hamzei Taj et al. 2016; $\mathrm{Xu}$ et al. 2016; Zhang et al. 2017). Microglia can change its morphology and express MHC-II, allowing them to function as antigen presenting cells that present neuronal debris as antigen to invade T cells (Yanuck 2019). In agreement with microglia activation, profound morphological changes and MHC-II upregulation occurred upon graft-versus-host disease induction (Mathew et al. 2020). M1 transformation was prevented through reducing the release of inflammatory factors of M1 phenotype TNF- $\alpha$, IL- 6 , and IL- $1 \beta$, and increasing the release of cytokines of M2 phenotype, while increasing the expressions of M2 markers (CD206 and Arg-1) in vivo was concomitant with the amelioration of cerebral injury and neurological functions deficits (Han et al. 2018). M1 and M2 can alleviate $\mathrm{ICH}$-induced inflammatory response by mediating microglia M2 polarization (Shi et al. 2016; Lan et al. 2017; Zhou et al. 2017). The natural product pinocembrin could reduce the number of M1 microglia without affecting M2 microglia, inhibit neuroinflammation and protect hemorrhagic brain (Lan et al. 2017). Shi et al. (Shi et al. 2016) also found that sinomenine could reduce ICH-induced inflammation by attenuating M1 microglia and promoting M2 microglia. In addition, regulatory $\mathrm{T}$ lymphocytes (Tregs) were proved to accelerate brain recovery after ICH through modulating microglia polarization toward M2 phenotype (Zhou et al. 2017).

Promoting microglia M2 polarization via targeting control will possibly become a new direction for the treatment of neurological diseases mediated by inflammation. MicroRNAs (miRNAs) are endogenous small RNAs with 18-25 nucleotides in length ( $\mathrm{Yu}$ et al. 2014). Some miRNAs can reduce ICH-induced brain injury by targeting different pathways (Yang et al. 2015; Xu et al. 2017). For example, inhibiting miR-27b could alleviate ICH-induced brain injury by promoting Nrf2/ARE pathway activation (Xu et al. 2017), and miR-233 was proved to reduce inflammatory response via responding to NLRP3 inflammasome after ICH (Yang et al. 2015). Recent research showed that CEBPA, which is a transcription factor that mediates the differentiation of pluripotent myeloid progenitor cells into mature granulocytes, could mediate microglia polarization (Yu et al. 2017), and that miR-124 attenuated ICH-induced inflammatory injury by increasing M2-polarized microglia through targeting CEBPA ( $\mathrm{Yu}$ et al. 2017). In addition, CEBPA was reported to be a target of miR-367 in the growth regulation of glioma cells (He et al. 2018).
Previous study indicated that miR-367 could also reduce the inflammatory response of microglia (Yuan et al. 2015). However, whether miR-367 alleviates ICH-induced inflammatory injury by promoting microglia $\mathrm{M} 2$ polarization via CEBPA has not been identified. Thus, in this study, we further explored the potential roles of miR-367 and CEBPA in ICH.

\section{Materials and Methods}

Specimen collection Thirty ICH patients (16 male and 14 female, aged from 38 to $55 \mathrm{y}$ old) treated by craniotomy in the First Affiliated Hospital of Zhengzhou University between Apr. 2018 and Oct. 2018 were enrolled. The patients were confirmed as having ICH by CT scan or MRI. Patients with traumatic brain injury, secondary brain hemorrhage due to the use of anticoagulant, cerebral vascular malformation hemorrhage, cancer, or other causes, and patients with severe liver and kidney diseases or lung infection were excluded from the study. ICH specimens and normal specimens (each about $1 \mathrm{~mm}^{3}$ ) were obtained from perihematoma and the tissue distant from hematoma during hematoma evacuation, respectively. The collected specimens were stored at $4^{\circ} \mathrm{C}$, and examined within $24 \mathrm{~h}$. The study was approved by the Ethics Committee of the First Affiliated Hospital of Zhengzhou University, and informed consent was signed by the participants.

Microglia isolation and culture The monocytes THP-1 cells were purchased from the American Type Culture Collection (ATCC, Manassas, VA). Microglia were isolated from the normal specimens and washed with Hank's balanced salt solution (HBSS, Gibco BRL, Waltham, MA) to remove meninges and visible blood vessels. Then the specimens were minced and incubated with $0.25 \%$ trypsin-EDTA solution in phosphate-buffered saline (PBS, Sigma-Aldrich, Billerica, MA) at room temperature for $1 \mathrm{~h}$. The suspension was filtered and centrifuged (at room temperature, $300 \times g$, for $5 \mathrm{~min}$ ) to isolate mixed glia cells. The isolated cells were then plated in a $75-\mathrm{cm}^{2}$ culture flask at $2 \times 10^{7}$ cells per flask in Dulbecco's modified Eagle's medium (DMEM, Sigma-Aldrich) containing $10 \%$ fetal bovine serum (FBS, Sigma-Aldrich) at $37^{\circ} \mathrm{C}$ with $5 \% \mathrm{CO}_{2}$. The medium was changed every $3 \mathrm{~d}$. After $12 \mathrm{~d}$, microglia adhered to the bottom were isolated and cultured in the same way as described above.

Microglia identification The cells were washed with $3 \mathrm{ml}$ of PBS and treated with $2 \mathrm{ml}$ of $4 \%$ paraformaldehyde at room temperature for $15 \mathrm{~min}$. Then, the cells were washed three times with $4 \mathrm{ml}$ of PBS, and incubated in blocking buffer (5\% serum, $0.1 \%$ Triton $\mathrm{X}-100$, in PBS) for 30 min. Next, the cells were incubated with $200 \mu \mathrm{l}$ of anti-CD11b antibody (ab133357, 1:100, Abcam, Cambridge, MA) and diamidino-2phenylindole (DAPI) in blocking buffer overnight at $4^{\circ} \mathrm{C}$ and 
washed three times with $200 \mu$ of blocking buffer. Finally, the cells were incubated with $200 \mu \mathrm{l}$ of secondary antibody in blocking buffer in the dark for $30 \mathrm{~min}$ at room temperature and washed with PBS three times. Microglias were then observed under a fluorescence microscope. Microglia with a purity of higher than $90 \%$ were used for the study.

Preparation of erythrocyte lysate Healthy blood samples were collected from healthy adults ( 8 male and 6 female, aged from 40 to $52 \mathrm{yr}$ old) in the First Affiliated Hospital of Zhengzhou University between Apr. 2018 and Oct. 2018. Informed consents for participation in the scientific research were signed by the participants. Single-cell suspensions of erythrocytes were prepared. One milliliter of red blood cell lysing solution was added into $1 \times 10^{5}$ erythrocytes to incubate the cells for $20 \mathrm{~min}$, and then the cells were centrifuged at $2000 \times \mathrm{g}$ for $10 \mathrm{~min}$. After that, the supernatants were used as erythrocyte lysate.

Cell treatment Microglia were collected and seeded into 24well tissue culture plates at a density of $3 \times 10^{5}$ cells/well and then incubated with $10 \mu$ lof erythrocyte lysate or PBS for $3 \mathrm{~d}$. Cytokine levels in the supernatants were determined by quantitative real-time PCR (qRT-PCR).

Transfection Erythrocyte lysate-treated microglia were randomly divided into 8 groups, namely, MC, M, IC, I, MC + vector, $\mathrm{MC}+\mathrm{CEBPA}, \mathrm{M}+$ vector, and $\mathrm{M}+\mathrm{CEBPA}$. Erythrocyte lysate-treated THP-1 monocytes were randomly divided into 4 groups, namely MC, M, IC, and I. Transfection was performed using Lipofectamine ${ }^{\circledR} 2000$ reagent (Thermo Fisher, Carlsbad, CA) following the manufacturer's instructions. In brief, $1 \times 10^{5}$ cells were plated in 24-well plates overnight for attachment. The next day, $5 \mu \mathrm{l}$ of transfection reagent was diluted in $50 \mu \mathrm{l}$ of Opti-MEM $^{\circledR}$ medium (Thermo Fisher, Carlsbad, CA), while $14 \mu \mathrm{g}$ of DNA (miR-367 mimic, inhibitor, mimic control, or inhibitor control) (Dharmacon, Inc. Chicago, IL) was diluted in $700 \mu$ of Opti-MEM $^{\circledR}$ medium. Then, $150 \mu$ l of diluted DNA was added to $150 \mu \mathrm{l}$ of diluted Lipofectamine ${ }^{\circledR} 2000$ reagent and incubated for $5 \mathrm{~min}$ at room temperature. Finally, $50 \mu \mathrm{l}$ of DNA-lipid complex was added to the cells. The sequences of mimic (M), inhibitor (I), mimic control (MC), and inhibitor control (IC) were 3'-UCUCAACGUAUAAUCGUUGUCA5', 5'-AGAGUUGCAUAUUAGCAACAGU-5', 5'UUUGUACUACACAAAAGUACUG-3', and 5'CAGUACUUUUGUGUAGUACAAA-3', respectively. To determine whether CEBPA was involved in miR-367-mediated microglia polarization, mimic control and vector, mimic control and CEBPA, mimic and vector, or mimic and CEBPA was cotransfected into microglia using Lipofectamine ${ }^{\circledR} 2000$ reagent. The plasmid vector used for CEBPA transfection was pcDNA3.1. RNA expression level and protein expression level were detected $48 \mathrm{~h}$ and $72 \mathrm{~h}$ after the transfection.
Quantitative real-time PCR Total RNA was isolated using Trizol reagent (Invitrogen, Carlsbad, CA) according to the manufacturer's instructions. In brief, $100 \mathrm{mg}$ of collected tissues were triturated in liquid nitrogen, added with $1 \mathrm{ml}$ Trizol reagent, and homogenized using a homogenizer. To determine the expressions of erythrocyte lysate-treated cells $\left(2.5 \times 10^{5}\right)$, the growth medium was removed, and $1 \mathrm{ml}$ of Trizol reagent was added to lyse the cells, and the lysate was pipetted to be homogenized. The treated tissues and cells were incubated for $5 \mathrm{~min}$ to fully dissociate the nucleoprotein complexes. Then, $0.2 \mathrm{ml}$ of chloroform was added to the cells and incubated for $3 \mathrm{~min}$. The samples were centrifuged for $15 \mathrm{~min}$ at $12,000 \times \mathrm{g}$ at $4^{\circ} \mathrm{C}$. The mixture was then separated into a lower red phenolchloroform phase, a middle phase, and a colorless upper aqueous phase. Contents in the colorless upper aqueous phase were transferred to a new tube by angling the tube at $45^{\circ}$, and $0.5 \mathrm{ml}$ of isopropanol was then added to the aqueous phase. After incubating for $10 \mathrm{~min}$, total RNA was isolated by centrifugation at $12,000 \times g$ at $4^{\circ} \mathrm{C}$ for $10 \mathrm{~min}$. Reverse transcription was performed using M-MLV Reverse Transcriptase System (Promega, Madison, WI). Quantitative real-time PCR with Light Cycler (Roche Diagnostics, Mannheim, Germany) and SYBR Green I in SYBRRT-PCR Kit (TaKaRa Biotechnology, Dalian, China) were used to detect mRNA expression. GAPDH served as an internal RNA control for Bcl-2, Bax, and CEBPA. Primers were purchased from Bio Asia Corp. (Shanghai, China), and the sequences were as follows: The Bcl-2 forward: 5'-TTCTTTGAGTTCGGTGGGGTC-3' and reverse: 5'-TGCATATTTGTTTGGGGCAGG-3'; The Bax forward: 5'-TCCACCAAGAAGCTGAGCGAG-3' and reverse: 5'-GTCCAGCCCATGATGGTTCT-3'; The CEBPA forward: 5'-GCGGGAACGCAACAACATC- $3^{\prime}$ and reverse: 5'-GTCACTGGTCAACTCCAGCAC-3'; The GAPHD forward: 5'-CATGGTCTACATGTTCCAGT-3' and reverse: 5'GGCTAAGCAGTTGGTGGTGC-3'. MiR-367 was detected using a miRNA RT kit (ABI) and Taq Man Universal PCR Master Mix (ABI) according to the manufacturer's instructions. U6 served as an internal control for miR-367. Primers were purchased from Bio Asia Corp. (Shanghai, China) and the sequences were as follows: MiR-367 forward: 5'-ACTG TTGCTAATATGCAACTC-3' and reverse: 5'-GAAC ATGTCTGCGTATCTC-3'; U6 forward: 5'-AGAG AAGATTAGCATGGCCCCTG-3' and reverse: 5'-ATCC AGTGCAGGGTCCGAGG-3'. QRT-PCR reactions were performed under the following conditions: at $50^{\circ} \mathrm{C}$ for $5 \mathrm{~min}$, at $94^{\circ} \mathrm{C}$ for $30 \mathrm{~s}, 40$ cycles at $94^{\circ} \mathrm{C}$ for $5 \mathrm{~s}$, and at $60^{\circ} \mathrm{C}$ for $30 \mathrm{~s}$. Threshold cycle value (CT) was calculated by the ${ }^{\Delta \Delta} \mathrm{CT}$ method, and the data were analyzed using Light Cycler Software 4.0 (Roche Diagnostics). The experiments were carried out in triplicate.

Western blotting Total protein was extracted from cultured cells and quantified using Trizol reagent (Invitrogen) 
following the protocols. Equal amounts of protein from different cells were separated by $10 \%$ SDS-PAGE and transferred to a nitrocellulose membrane (Bio-Rad, Hercules, CA). The membrane was blocked with 5\% non-fat milk and incubated with antibodies against MHCII (ab55152, Abcam), IL-1 $\beta$ (\#12242, CST), CD206 (ab64693, Abcam), Arg-1 (\#9819, CST), GAPDH (ab8245, Abcam), CEBPA (ab40764, Abcam), and $\beta$ actin (ab8245, Abcam) at the concentration of 1:1000 at $4^{\circ} \mathrm{C}$ overnight. The blots were then incubated with horseradish peroxidase-conjugated secondary antibodies in blocking buffer at room temperature for $2 \mathrm{~h}$. The signal was detected using ECL system (Amersham Pharmacia) and quantified by scanning densitometry and computerassisted image analyzer.

Flow cytometry assay of apoptosis Apoptotic cells were quantified by Annexin V-FITC-propidium iodide (PI) double staining using an Annexin V-FITC apoptosis detection kit. The cells were washed twice with PBS and diluted to a density of $1 \times 10^{6}$ cells $/ \mathrm{ml}$. Ten microliters of Annexin V-FITC and $10 \mu \mathrm{l}$ of PI $(20 \mu \mathrm{g} / \mathrm{ml})$ were added into $100 \mu \mathrm{l}$ of suspensions and incubated for at least $20 \mathrm{~min}$ at room temperature in the dark. Four hundred microliters of PBS binding buffer was then added to each tube. The cells were analyzed using FCM analysis (BD Biosciences Clontech) and CellQuest Pro software version 5.1.

Dual-luciferase reporter gene assay The cells at $70-80 \%$ confluence were co-transfected in 24-well plates. Then, $0.3 \mu \mathrm{g}$ of reporter gene plasmid, $0.02 \mu \mathrm{g}$ of internal control vector pGL4.74 [hRluc/TK] vector (Promega, Fitchburg, WI), $1 \mu \mathrm{l}$ of transfection agent, and $0.2 \mu \mathrm{g}$ of expression vector orsi-RNA were mixed together. Fortyeight hours after the transfection, dual-luciferase reporter assay was performed according to the manufacturer's instructions. The cells were lysed using $1 \times$ reporter lysis buffer and harvested. Luminescence was detected by Mithras LB 940 (Berthold Technologies, Oak Ridge, $\mathrm{TN})$. The firefly luciferase activity of the reporter gene plasmid was measured as $1(\mathrm{~m} 1)$, while the renilla luciferase activity (internal control) of pGL4.74 [hRluc/TK] vector was measured as $2(\mathrm{~m} 2)$. The relative luciferase activity was calculated by the ratio of $\mathrm{m} 1 / \mathrm{m} 2$.

Statistical analysis The data were analyzed using SPSS version 16.0 (SPSS Inc., Chicago, IL) and shown as means \pm standard errors of the means. Statistical analysis was performed by one-way analysis of variance (ANOVA), followed by Dunnett's post hoc test. $p<0.05$ was considered statistically significant.

\section{Results}

MiR-367 expression was downregulated in perihematoma of ICH patients QRT-PCR was performed to detect the expression level of miR-367 in ICH specimens and normal specimens. As shown in Fig. 1 $a$, compared with normal specimens, the level of miR-367 in ICH specimens was reduced significantly $(p<0.01)$.

Microglia identification Microglia were isolated to establish the ICH cell culture model. Immunocytochemistry was performed using CD11b as a marker of microglia and DAPI as a marker of cell nucleus to identify isolated microglia. The mergence showed that microglia and cell nuclei could perfectly correspond to each other (Fig. 1B), suggesting that the culture isolated was pure microglia. As some cells were in different division stages with different brightness, so some cells which were brighter than the others, which might be a limitation.

\section{Erythrocyte lysate-treated microglias were established} Erythrocyte lysate-treated microglias were established ( $\mathrm{Yu}$ et al. 2017) to verify the specific change of miR-367 level. First, we found that erythrocyte lysate significantly increased the protein expressions of M1 microglia markers (MHC-II and IL-1 $\beta$ ) and reduced those of M2 microglia markers (CD206 and Arg-1) (Fig. 1c, d). Then, the levels of miRNAs were detected by qRT-PCR. Compared with the PBS-treated group, the level of miR-367 in erythrocyte lysate-treated microglia was significantly decreased, the level of miR-124 slightly decreased, and miR-155 slightly

Figure 1. The effects of ICH, erythrocyte lysate, and up/downregulation miR-367 on the mRNA expression level of miR-367. (a) The change of miR-367 expression in perihematoma of patients with ICH was determined by qRT-PCR (*vs. normal; **p<0.01). (b) Immunofluorescence of isolated microglia probing for CD11b in gree channel and DAPI in blue channel. $(c, d)$ The protein levels of M1 microglia markers (MHC-II and IL-1 $\beta$ ) and M2 microglia markers (CD206 and Arg-1) were detected by Western blotting assay (*vs. PBS; $* * p<0.01$ ). (e) QRT-PCR for miRNAs from microglia treated with erythrocyte lysate or PBS for $3 \mathrm{~d}$ was applied (*vs. PBS; ** $p<0.01)$. $(f)$ QPCR was used to detect the effects of mimic and inhibitor on the mRNA expression level of miR-367 $\left({ }^{*}\right.$ vs. MC; ${ }^{\#}$ vs. IC; $\left.{ }^{* * \# \#} p<0.01\right) .(g, h)$ The protein levels of M1 microglia markers (MHC-II and IL-1 $\beta$ ) and M2 microglia markers (CD206 and Arg-1) were detected by Western blotting assay. (i) QPCR was used to detect the effects of mimic and inhibitor on the mRNA expression level of miR-367 in THP-1 cells (*vs. MC; ${ }^{\#}$ vs. IC; $\left.{ }^{*}{ }^{\prime \# \#} p<0.01\right) .(j, k)$ The protein levels of M1 microglia markers (MHC-II and IL-1 $\beta$ ) and M2 microglia markers (CD206 and Arg-1) were detected by Western blotting assay in THP- 1 cells. Data were shown as mean \pm SD from three independent experiments and analyzed by Dunnett's $t$ test. ICH, intracerebral hemorrhage; qRT-PCR, quantitative real-time polymerase chain reaction; DAPI, 4',6-diamidino-2-phenylindole; PBS, phosphate-buffered solution; MC, mimic control; M, mimic; IC, inhibitor control; I, inhibitor; $\mathrm{SD}$, standard deviation 


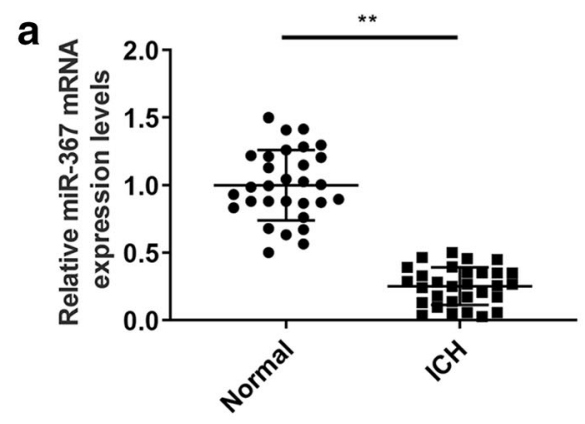

b

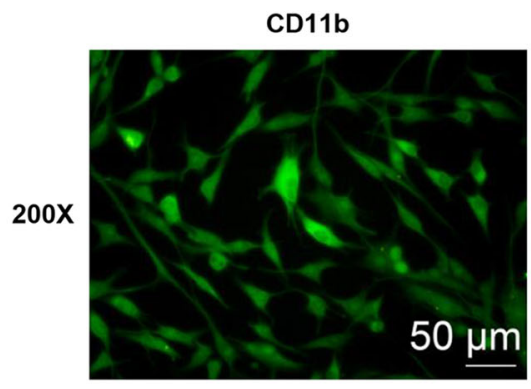

Dapi

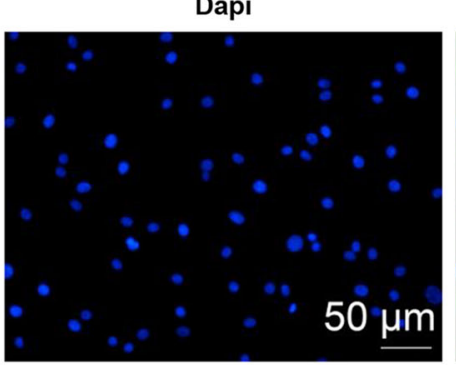

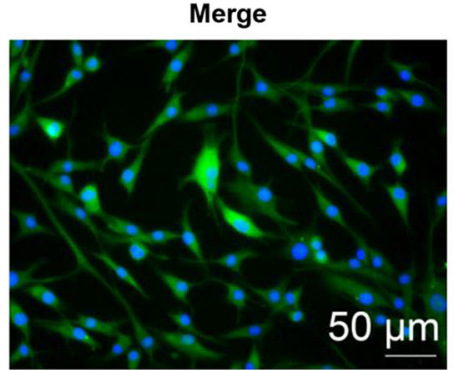

e

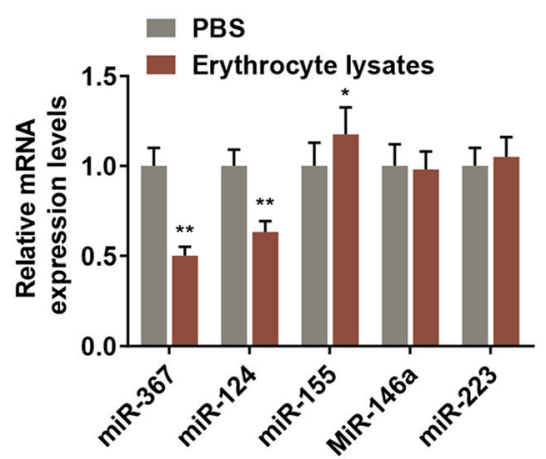

h

g
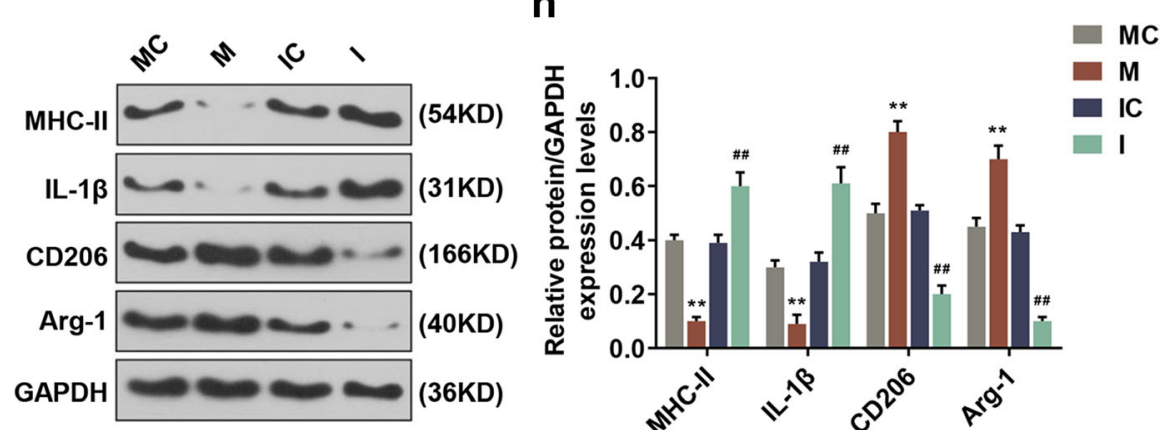

k

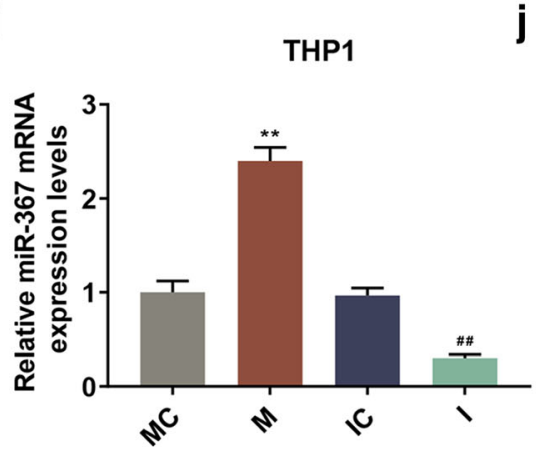

THP1

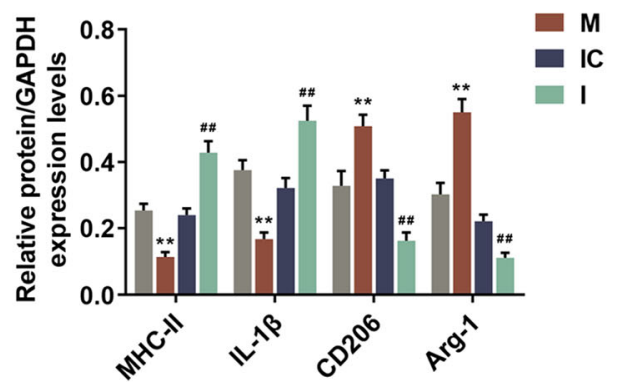



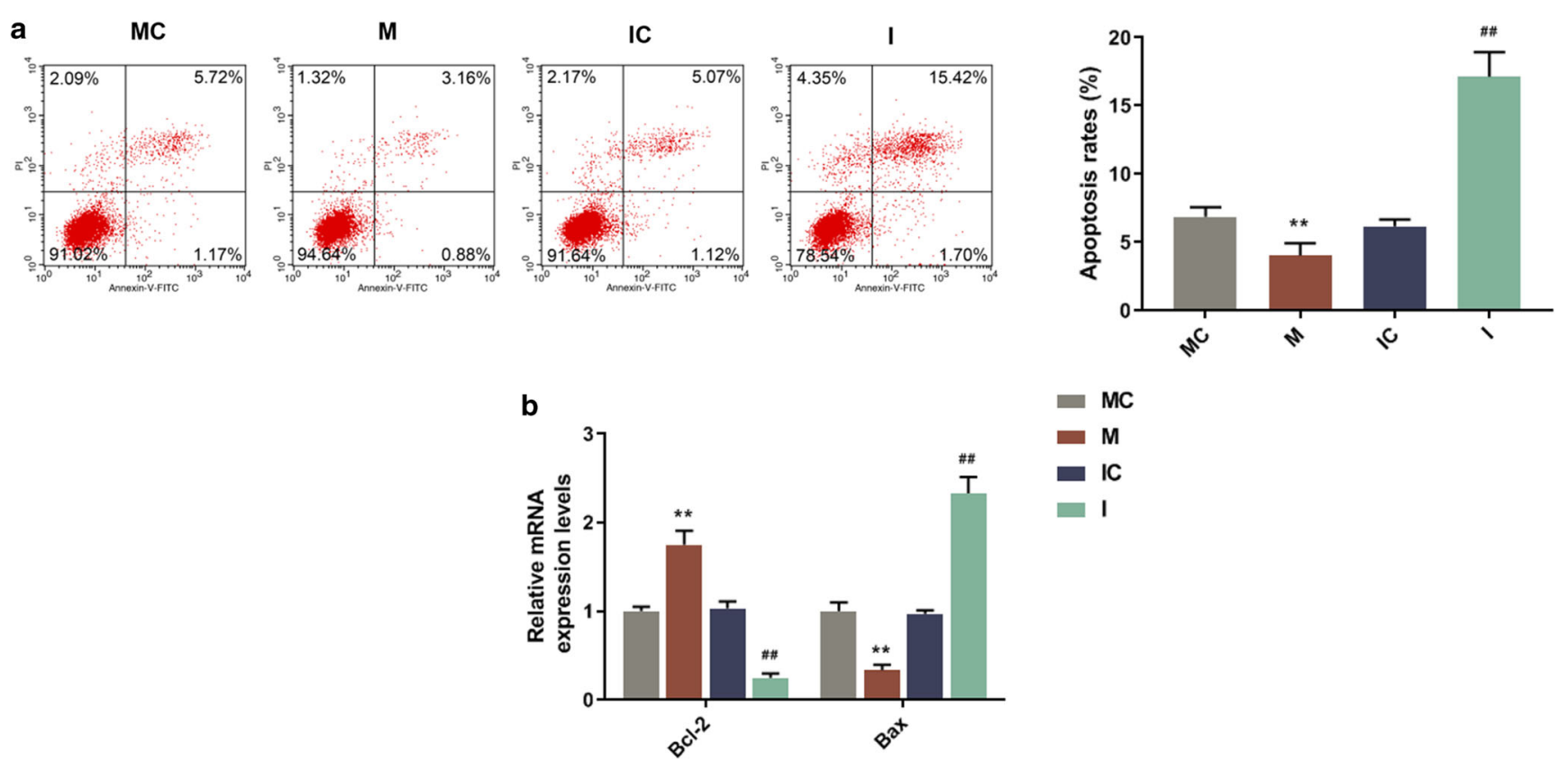

Figure 2. The effects of up/downregulated miR-367 on microglia polarization and cell apoptosis. (a) Flow cytometry was used to assess the effect of miR-367 mimic or inhibitor on the apoptosis of erythrocyte lysate-treated microglia. (b) QRT-PCR was performed to determine the effect of miR-367 mimic or inhibitor on the mRNA expression levels of

increased, while the level of miR-146a and miR-223 changed a little (Fig. 1e). The change of miR-367 was the highest among detected miRNAs and was consistent with the results in ICH patients, suggesting that erythrocyte lysate-treated microglia were successfully established.

\section{MiR-367 mimic promoted microglia M2 polarization and re-} duced apoptosis in erythrocyte lysate-treated microglia To further explore the function of miR-367 in microglia polarization and apoptosis in erythrocyte lysate treated microglia, mRNA level, protein expression and cell apoptosis were determined by qRT-PCR, Western blotting and flow cytometry, respectively. MiR-367 mimic and inhibitor were used to upregulate and downregulate miR-367 in erythrocyte lysatetreated microglia (Fig. 1f), respectively. We found that miR367 mimic significantly reduced the protein expressions of M1 microglia markers (MHC-II and IL-1 $\beta$ ) and increased those of M2 microglia markers (CD206 and Arg-1) (Fig. 2g, $h$ ), indicating that overexpressed miR-367 promoted microglia M2 polarization. Other than microglia, miR-367 mimic and inhibitor were used to upregulate and downregulate miR-367 in erythrocyte lysate-treated THP-1 monocytes (Fig. 1i), respectively. We found that miR-367 mimic significantly reduced the protein expressions of M1 microglia markers (MHC-II and IL-1 $\beta$ ) and increased those of M2 microglia markers (CD206 and Arg-1) in erythrocyte lysate-treated THP-1 monocytes (Fig. 2j, $k$ ), indicating that overexpressed miR-367 promoted M2 polarization in THP-1 monocytes.
Hence, the results indicated that overexpressed miR-367 promoted microglia M2 polarization.

Moreover, miR-367 mimic could also upregulate the level of Bcl-2, downregulate the level of Bax, and reduce apoptosis rate (Fig. 2a,b), while miR-367 inhibitor exerted an opposite effect to that of miR-367 mimic in erythrocyte lysate-treated microglia.

CEBPA was a direct target for miR-367 in microglia Target Scan 7.2 (Fig. $3 a$ ) predicted that CEBPA is a potential target for miR-367, and the direct relationship between CEBPA and miR-367 was further confirmed by dual luciferase reporter system. We found that co-transfecting with miR-367 mimic and wild-type CEBPA 3'-UTR greatly suppressed the luciferase expression level. However, co-transfecting with miR-367 mimic and mutated CEBPA 3'-UTR did not affect the luciferase expression level (Fig. 3b). These results demonstrated that CEBPA could be suppressed by miR-367.

MiR-367 mimic downregulated CEBPA expression in erythrocyte lysate-treated microglia To explore the effect of miR367 mimic on the expression of CEBPA in erythrocyte lysatetreated microglia, Western blotting and qRT-PCR assays were performed to detect the protein and mRNA expression levels of CEBPA. We found that transduction of miR-367 mimic downregulated the mRNA and protein expression levels of CEBPA (Fig. $3 c-e$ ). 

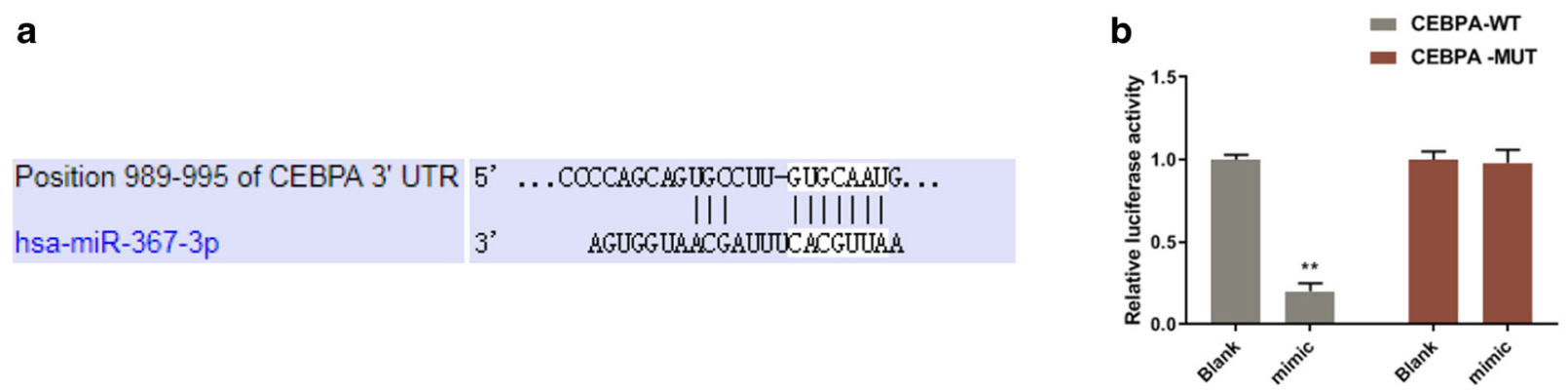

c

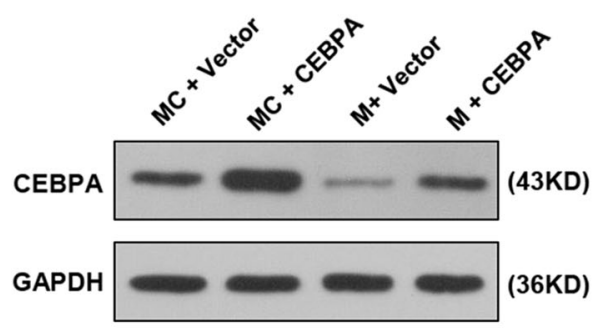

d

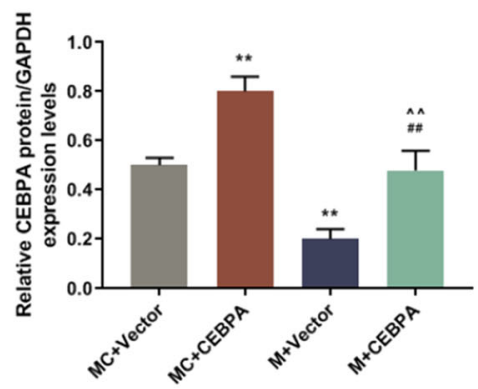

e

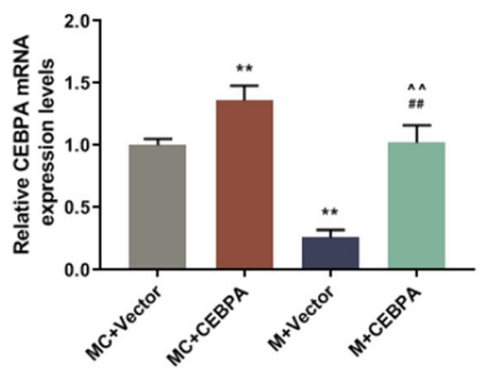

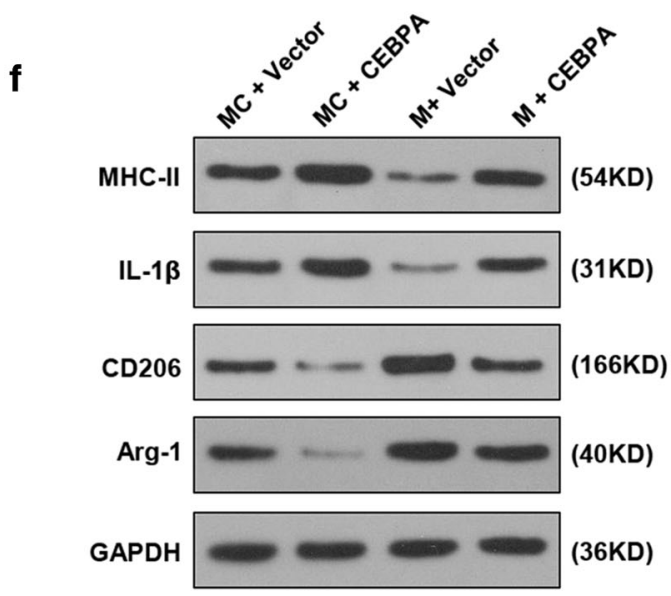

Figure 3. CEBPA was a direct target for miR-367, and miR-367 promoted microglia M2 polarization via CEBPA. (a) Sequences of hsa-miR$367-3 p$ recognition region predicted by Targetscan7.2 in the 3 '-UTR of CEBPA. (b) Luciferase activities of reporter plasmids with wild type or mutated CEBPA 3 '-UTR in microglia were examined by transfecting mimics or none $(* v s$. blank; $* * p<0.01)$. $(c, d)$ Detection of CEBPA protein levels in microglia by Western blotting $\left({ }^{*} \mathrm{vs} . \mathrm{MC}+\right.$ vector; ${ }^{*} \mathrm{vs}$. $\mathrm{M}+$ vector; $\left.*^{* / \# \#} p<0.01\right)$. (e) QRT-PCR was used to determine the

MiR-367 mimic promoted microglia M2 polarization and reduced apoptosis through CEBPA in erythrocyte lysate-treated microglia To determine the effects of CEBPA on microglia treated with miR-367 mimic, Western blotting and flow cytometry assays were performed to determine protein expression and cell apoptosis. Obviously, over-expression of miR367 significantly improved M2 polarization in erythrocyte lysate-treated microglia, while co-transduction with CEBPA produced no effect (Fig. $3 f$, g). Additionally, CEBPA inhibited the reduction of apoptosis rate caused by miR-367 g

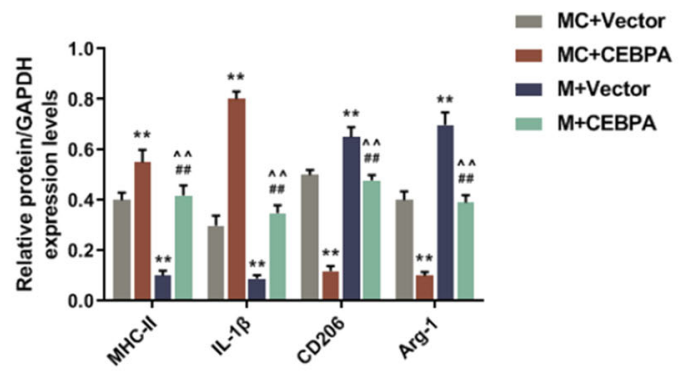

effects of CEBPA and mimic on the mRNA expression level of CEBPA (*vs. MC + vector; ${ }^{\#}$ vs. $\mathrm{M}+$ vector; $\left.* * / \# p<0.01\right) .(f, g)$ The protein levels of M1 microglia markers (MHC-II and IL-1 $\beta$ ) and M2 microglia markers (CD206 and Arg-1) detected by Western blotting assay $\left(*\right.$ vs. $\mathrm{MC}+$ vector; ${ }^{\#}$ vs. $\mathrm{M}+$ vector; ${ }^{\wedge}$ vs. $\mathrm{MC}+\mathrm{CEBPA}$; $\left.* * *^{* \# / \wedge} p<0.01\right)$. Data were shown as mean $\pm \mathrm{SD}$ from three independent experiments and analyzed by Dunnett's $t$ test.

(Fig. 4). These data demonstrated that over-expression of miR-367 promoted microglia M2 polarization and reduced apoptosis via downregulating CEBPA.

\section{Discussion}

ICH is one of the most acute and serious types of stroke. However, there is a lack of a specific treatment for $\mathrm{ICH}$ (Hamzei Taj et al. 2016). Intensive blood pressure reduction 


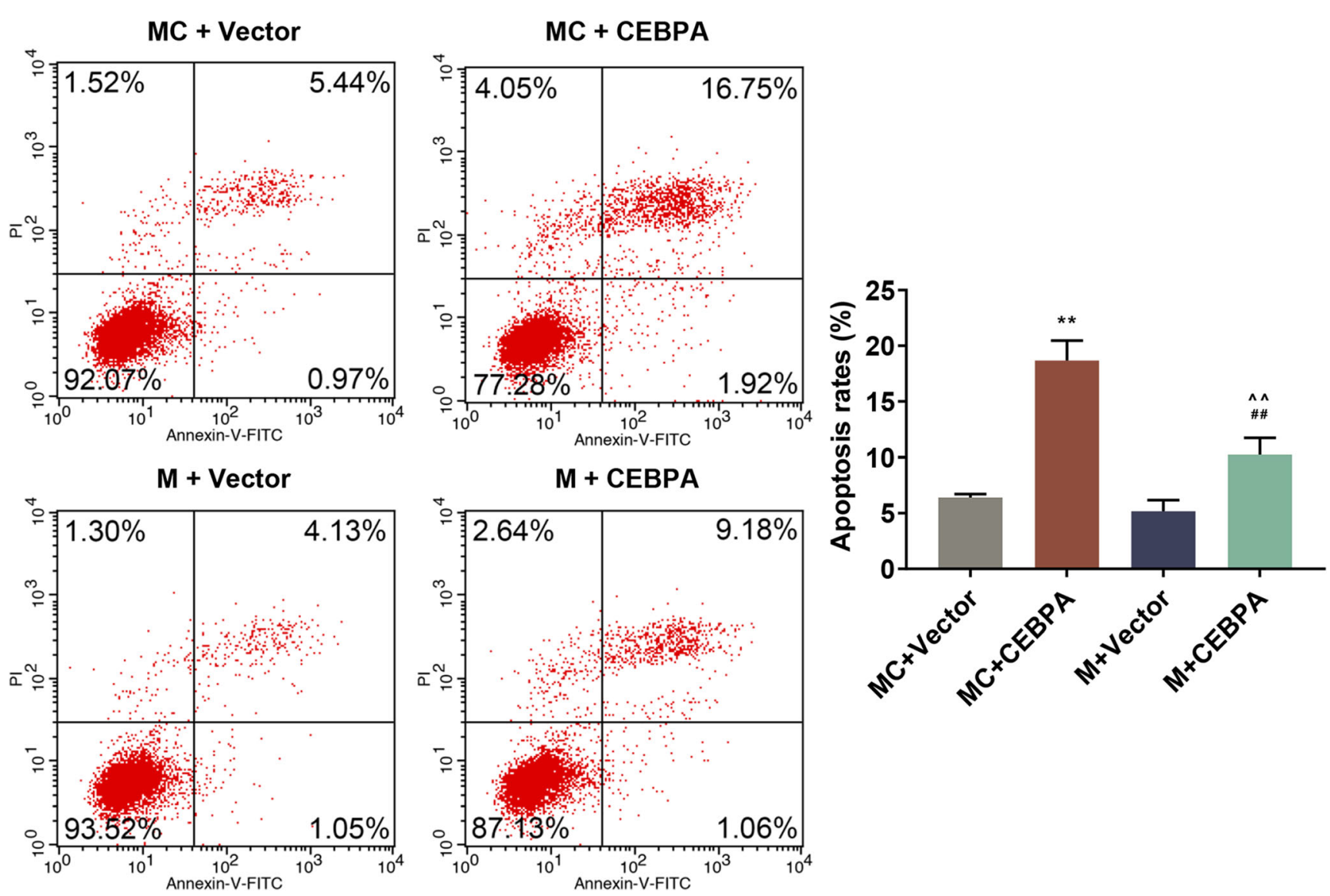

Figure 4. The effects of CEBPA and mimic on microglia apoptosis assessed by flow cytometry. Data were shown as mean $\pm \mathrm{SD}$ from three independent experiments and analyzed using Dunnett's $t$ test $\left({ }^{*}\right.$ vs. $\mathrm{MC}+$ vector; ${ }^{\#} \mathrm{vs} . \mathrm{M}+$ vector; $\left.{ }^{\wedge} \mathrm{vs} . \mathrm{MC}+\mathrm{CEBPA} ; *^{*} p<0.05 ; * * / \# \# / \wedge \wedge<0.01\right)$.

and hemostatic therapy could possibly attenuate ICH-induced brain injury (Wartenberg and Mayer 2015; Morotti et al. 2017). Evacuation of hematoma by surgical treatment is another approach to alleviate ICH injury (Mendelow 2015; Rennert et al. 2015). Moreover, as secondary injury also contributes to brain injury after ICH (Lee et al. 2016), reducing secondary injury is equally important in treating ICH. Studies showed that the process of nerve cell injury after ICH is affected by secondary ischemia in peripheral brain tissue, thrombin release, hemoglobin toxicity, inflammatory response, and apoptosis, and among the above factors, inflammatory response is one of the most important affecting factors (Zhang et al. 2017). Numerous reports indicated that the reduction of inflammatory injury had a positive effect on the prognosis of ICH (Lee et al. 2016; Yu et al. 2017; Zhang et al. 2017).

Microglia are inflammatory cells (Shi et al. 2016), and specifically, M1 microglia increase inflammation via producing pro-inflammatory cytokines (e.g., MHC-II, IL-1 $\beta$ ), while M2 microglia reduce inflammation by releasing neuroprotective factors (e.g., CD206, Arg-1) (Lee et al. 2016; Yu et al. 2017) (Yuan et al. 2015). Therefore, mediating microglia M2 polarization could be explored to treat $\mathrm{ICH}$ patients. Studies showed that miRNA expression level changed with microglia phenotypes, and that miRNAs also regulated the polarizaion of microglia (Yu et al. 2014; Yang et al. 2015; Xu et al. 2017).

MicroRNAs (miRNAs) are endogenous small RNAs with 18-25 nucleotides in length (Yu et al. 2014), and they can regulate microglia polarization via various pathways. MiR155 is an M1-related miRNA, and it was reported that miR155 could affect the interleukin 13-dependent regulation of several genes (SOCS1, DC-SIGN, CCL18, CD23, and SERPINE), thereby affecting the establishment of an M2 phenotype in macrophages (Martinez-Nunez et al. 2011). MiR146 acts as an anti-inflammatory miRNA to target TLR4 signaling and thereby suppresses iNOs and promotes M2 polarization (Vergadi et al. 2014). MicroRNA-223 regulates inflammation and brain injury via feedback to NLRP3 inflammasome after intracerebral hemorrhage (Yang et al. 2015). MiR-124 was shown to ameliorate ICH-induced inflammatory injury via promoting microglia M2 polarization through CEBPA (Yu et al. 2017). 
CEBPA has been proved as a tumor-inhibiting factor and is related to various types of cancers (Lourenco and Coffer 2017; Voutila et al. 2017). Upregulation of CEBPA might reduce key pro-inflammatory cytokines, thus showing an antiinflammatory potential (Freire and Conneely 2018; Zhou et al. 2019). It was also reported that CEBPA mediated microglia polarization and could be beneficial in reducing ICHinduced inflammatory injury [13]. Recent research indicated that miR-367 could downregulate the inflammatory response of microglia (Yuan et al. 2015). Similarly, it was found that miR-367 expression was decreased in perihematoma of patients with ICH in our study. MiR-367 belonged to miR-92a family and it was reported barely detectable in macrophages (Lai et al. 2013). To investigate the mechanisms of miR-367 and CEBPA in ICH, cell culture of erythrocyte lysate-treated microglia was successfully established by erythrocyte lysate treatment, which was confirmed by the increase of M1 microglia markers (MHC-II and IL-1 $\beta$ ) and decrease of M2 microglia markers (CD206 and Arg-1). The representative miRNAs (miR-367, miR-124, miR-155, miR-146a, miR-223) being related to M1/M2 was detected, and miR-367 level was found to significantly decrease by erythrocyte lysate treatment. Then, we found that upregulating miR-367 could significantly decrease the protein expressions of M1 microglia markers (MHC-II and IL-1 $\beta$ ) and increase those of M2 microglia markers (CD206 and Arg-1) in erythrocyte lysate-treated microglias and monocytes. Cell apoptosis was a common mechanism in many bio-activities (Zhang et al. 2019). Moreover, we found that miR-367 mimic could also upregulate $\mathrm{Bcl}-2$, downregulate $\mathrm{Bax}$, and reduce apoptosis rate. However, miR-367 inhibitor exerted an opposite effect to that of miR-367 mimic. CEBPA was predicted as a direct target for miR-367 in microglia, and it could promote microglia M1 polarization (MHC-II and IL-1 $\beta$ ), inhibit microglia M2 polarization (CD206 and Arg-1), and increase apoptosis rate. The effect of CEBPA on inflammatory factors in our study was opposite to the observations before (Freire and Conneely 2018), which might depend on different mechanisms. However, when CEBPA and miR-367 co-existed, the protein and mRNA expressions of CEBPA were decreased, resulting in a reduction of microglia $\mathrm{M} 1$ polarization, an increase of microglia M2 polarization, and a lower apoptosis rate. MHCII, IL-1 $\beta$, CD206, and Arg-1 studied in our research are representative factors that reflect microglia M1 and M2 polarization types. More specific high molecular markers for microglia M1 and M2 polarization types could be studied in the future. In addition, more in vivo experiments are needed in future study to further verify the results. Besides, we did not study humoral molecules from erythrocytes or the molecular interaction between humoral molecules and microglia nor did we apply monocytes or macrophages to compare with the effect of miR-367 on microglia or even compare miR-367 with other miRNAs. These works would be conducted in the future.
In conclusion, our findings demonstrated that CEBPA aggravated inflammatory injury caused by erythrocyte lysate, while miR-367 could attenuate the injury by promoting microglia M2 polarization via targeting downregulated CEBPA. The results of our study provide a new feasible strategy for alleviating secondary injury in ICH.

Funding This work was supported by the Innovation Foundation of Youth in the First Affiliated Hospital of Zhengzhou University. Thanks for the financial support.

\section{Compliance with ethical standards}

Conflict of interest The authors declare that they have no conflict of interest.

Open Access This article is licensed under a Creative Commons Attribution 4.0 International License, which permits use, sharing, adaptation, distribution and reproduction in any medium or format, as long as you give appropriate credit to the original author(s) and the source, provide a link to the Creative Commons licence, and indicate if changes were made. The images or other third party material in this article are included in the article's Creative Commons licence, unless indicated otherwise in a credit line to the material. If material is not included in the article's Creative Commons licence and your intended use is not permitted by statutory regulation or exceeds the permitted use, you will need to obtain permission directly from the copyright holder. To view a copy of this licence, visit http://creativecommons.org/licenses/by/4.0/.

\section{References}

Boche D, Perry VH, Nicoll JA (2013) Review: activation patterns of microglia and their identification in the human brain. Neuropathol Appl Neurobiol 39:3-18. https://doi.org/10.1111/nan.12011

Freire PR, Conneely OM (2018) NR4A1 and NR4A3 restrict HSC proliferation via reciprocal regulation of $\mathrm{C} / \mathrm{EBPalph}$ and inflammatory signaling. Blood 131:1081-1093. https://doi.org/10.1182/blood2017-07-795757

Hamzei Taj S et al (2016) Dynamic modulation of microglia/macrophage polarization by miR-124 after focal cerebral ischemia. J NeuroImmune Pharmacol 11:733-748. https://doi.org/10.1007/ s11481-016-9700-y

Han $\mathrm{Z}$ et al (2018) TOPK promotes microglia/macrophage polarization towards M2 phenotype via inhibition of HDAC1 and HDAC2 activity after transient cerebral ischemia. Aging Dis 9:235-248. https://doi.org/10.14336/ad.2017.0328

He Y, Zhou L, Fan Z, Liu S, Fang W (2018) Palmitic acid, but not highglucose, induced myocardial apoptosis is alleviated by $\mathrm{N}$ acetylcysteine due to attenuated mitochondrial-derived ROS accumulation-induced endoplasmic reticulum stress. Cell Death Dis 9:568. https://doi.org/10.1038/s41419-018-0593-y

Lai L et al (2013) MicroRNA-92a negatively regulates toll-like receptor (TLR)-triggered inflammatory response in macrophages by targeting MKK4 kinase. J Biol Chem 288:7956-7967. https://doi. org/10.1074/jbc.M112.445429

Lan X et al (2017) Pinocembrin protects hemorrhagic brain primarily by inhibiting toll-like receptor 4 and reducing M1 phenotype microglia. Brain Behav Immun 61:326-339. https://doi.org/10.1016/j.bbi. 2016.12.012 
Lee JH et al (2016) Regulation of therapeutic hypothermia on inflammatory cytokines, microglia polarization, migration and functional recovery after ischemic stroke in mice. Neurobiol Dis 96:248-260. https://doi.org/10.1016/j.nbd.2016.09.013

Lourenco AR, Coffer PJ (2017) A tumor suppressor role for C/EBPalpha in solid tumors: more than fat and blood. Oncogene 36:5221-5230. https://doi.org/10.1038/onc.2017.151

Martinez-Nunez RT, Louafi F, Sanchez-Elsner T (2011) The interleukin 13 (IL-13) pathway in human macrophages is modulated by microRNA-155 via direct targeting of interleukin 13 receptor alpha1 (IL13Ralpha1). J Biol Chem 286:1786-1794. https://doi.org/10. 1074/jbc.M110.169367

Mathew NR et al (2020) Graft-versus-host disease of the CNS is mediated by TNF upregulation in microglia. J Clin Invest 130:13151329. https://doi.org/10.1172/jci130272

Mendelow AD (2015) Surgical craniotomy for intracerebral haemorrhage. Front Neurol Neurosci 37:148-154. https://doi.org/10.1159/ 000437119

Miron VE et al (2013) M2 microglia and macrophages drive oligodendrocyte differentiation during CNS remyelination. Nat Neurosci 16: 1211-1218. https://doi.org/10.1038/nn.3469

Morotti A, Brouwers HB, Romero JM, Jessel MJ, Vashkevich A, Schwab K, Afzal MR, Cassarly C, Greenberg SM, Martin RH, Qureshi AI, Rosand J, Goldstein JN, for the Antihypertensive Treatment of Acute Cerebral Hemorrhage II and Neurological Emergencies Treatment Trials Investigators (2017) Intensive blood pressure reduction and spot sign in intracerebral hemorrhage: a secondary analysis of a randomized clinical trial. JAMA Neurol 74:950-960. https://doi.org/10.1001/jamaneurol.2017.1014

Psaila B, Petrovic A, Page LK, Menell J, Schonholz M, Bussel JB (2009) Intracranial hemorrhage (ICH) in children with immune thrombocytopenia (ITP): study of 40 cases. Blood 114:4777-4783. https://doi. org/10.1182/blood-2009-04-215525

Rennert RC, Signorelli JW, Abraham P, Pannell JS, Khalessi AA (2015) Minimally invasive treatment of intracerebral hemorrhage. Expert Rev Neurother 15:919-933. https://doi.org/10.1586/14737175. 2015.1059755

Shi H et al (2016) Sinomenine enhances microglia M2 polarization and attenuates inflammatory injury in intracerebral hemorrhage. J Neuroimmunol 299:28-34. https://doi.org/10.1016/j.jneuroim. 2016.08.010

Tatlisumak T, Cucchiara B, Kuroda S, Kasner SE, Putaala J (2018) Nontraumatic intracerebral haemorrhage in young adults. Nat Rev Neurol 14:237-250. https://doi.org/10.1038/nrneurol.2018.17

van Asch CJ, Luitse MJ, Rinkel GJ, van der Tweel I, Algra A, Klijn CJ (2010) Incidence, case fatality, and functional outcome of intracerebral haemorrhage over time, according to age, sex, and ethnic origin: a systematic review and meta-analysis. Lancet Neurol 9:167-176. https://doi.org/10.1016/s1474-4422(09)70340-0

Vergadi E et al (2014) Akt2 deficiency protects from acute lung injury via alternative macrophage activation and miR-146a induction in mice. J Immunol 192:394-406. https://doi.org/10.4049/jimmunol. 1300959
Voutila J et al (2017) Development and mechanism of small activating RNA targeting CEBPA, a novel therapeutic in clinical trials for liver cancer. Mol Ther 25:2705-2714. https://doi.org/10.1016/j.ymthe. 2017.07.018

Wartenberg KE, Mayer SA (2015) Ultra-early hemostatic therapy for intracerebral hemorrhage: future directions. Front Neurol Neurosci 37:107-129. https://doi.org/10.1159/000437117

Xu W, Li F, Liu Z, Xu Z, Sun B, Cao J, Liu Y (2017) MicroRNA-27b inhibition promotes Nrf2/ARE pathway activation and alleviates intracerebral hemorrhage-induced brain injury. Oncotarget 8: 70669-70684. https://doi.org/10.18632/oncotarget.19974

Xu X, Gao Y, Wen L, Zhai Z, Zhang S, Shan F, Feng J (2016) Methionine enkephalin regulates microglia polarization and function. Int Immunopharmacol 40:90-97. https://doi.org/10.1016/j. intimp.2016.08.037

Yang Z, Zhong L, Xian R, Yuan B (2015) MicroRNA-223 regulates inflammation and brain injury via feedback to NLRP3 inflammasome after intracerebral hemorrhage. Mol Immunol 65: 267-276. https://doi.org/10.1016/j.molimm.2014.12.018

Yanuck SF (2019) Microglial phagocytosis of neurons: diminishing neuronal loss in traumatic, infectious, inflammatory, and autoimmune CNS disorders. Front Psychiat 10:712. https://doi.org/10.3389/ fpsyt.2019.00712

Yu A et al (2017) MiR-124 contributes to M2 polarization of microglia and confers brain inflammatory protection via the C/EBP-alpha pathway in intracerebral hemorrhage. Immunol Lett 182:1-11. https://doi.org/10.1016/j.imlet.2016.12.003

Yu H, Lu Y, Li Z, Wang Q (2014) microRNA-133: expression, function and therapeutic potential in muscle diseases and cancer. Curr Drug Targets 15:817-828

Yuan B, Shen H, Lin L, Su T, Zhong L, Yang Z (2015) MicroRNA367 negatively regulates the inflammatory response of microglia by targeting IRAK4 in intracerebral hemorrhage. J Neuroinflammation 12:206. https://doi.org/10.1186/s12974-0150424-3

Zhang H, Wu D, Jin M (2019) GCDH contributes to better outcome and acts on chemoresistance and immune exclusion in cervical cancer. Eur J Gynaecol Oncol 40:831-838. https://doi.org/10.12892/ ejgo4902.2019

Zhang Z, Zhang Z, Lu H, Yang Q, Wu H, Wang J (2017) Microglial polarization and inflammatory mediators after intracerebral hemorrhage. Mol Neurobiol 54:1874-1886. https://doi.org/10.1007/ s12035-016-9785-6

Zhou J et al (2019) Anti-inflammatory activity of MTL-CEBPA, a small activating RNA drug, in LPS-stimulated monocytes and humanized mice. Mol Ther doi:https://doi.org/10.1016/j.ymthe.2019.02.018

Zhou K et al (2017) Regulatory T cells ameliorate intracerebral hemorrhage-induced inflammatory injury by modulating microglia/macrophage polarization through the IL-10/GSK3beta/ PTEN axis. J Cereb Blood Flow Metab 37:967-979. https://doi. org/10.1177/0271678x16648712 